\title{
Proyecto Portal Bicentenario
}

\section{Portal Bicentenario project}

Sergio Galilea 0.

$<$ Resumen>

En este artículo el actual gerente del proyecto "Portal Bicentenario" presenta algunos procesos y obras que comienzan a dar forma al Portal Bicentenario.

$<$ Abst ract $>$

The current manager of the "Portal Bicentenario Project" presents some of the processes and works that are giving shape to this project.

<Palabras clave>
RENOVACIÓN URBANA / PARQUES URBANOS /

BICENTENARIO INDEPENDENCIA DE CHILE (2010)/ REUTILIZACIÓN DE INFRAESTRUCTURAS DE TRANSPORTE (AEROPUERTOS) / CERRILLOS, SANTIAGO

\section{$<$ Key words>}

URBAN RENOVATI ON / URBAN PARKS / BI CENTENNI AL OF THE I NDEPENDENCE OF CHI LE (2010) / RECYCLI NG OF TRANSPORT I NFRASTRUCTURE ( AI RPORTS) CERRI LLOS, SANTI AGO, CHI LE

\section{Introducción}

El proyecto Portal Bicentenario busca transformarse en un ejemplo emblemático, respecto a la generación de condiciones urbanas apropiadas para la consolidación del proceso de reconversión de los vacíos interiores urbanos. En este escenario, el terreno del ex aeródromo Los Cerrillos presenta un especial desafío debido a sus condiciones físicas, de localización y presencia en la memoria colectiva de la ciudad. Al mismo tiempo, ofrece la oportunidad de repensar el valor y el potencial de un trozo de ciudad, desde la perspectiva de un desarrollo urbano integrador, integral y amable con su medio ambiente.

Se pretende desarrollar ciudad "en el interior de la ciudad", aprovechando la actual capacidad instalada de un lote extenso y de uso libre urbano, "localizado" adecuadamente en el Gran Santiago, con buena relación con la red vial estructurante, dotado de un conjunto de servicios esenciales y cercano a áreas de actividad laboral y de servicios. El proyecto aspira a albergar en 20 años a 15.000 familias, en un barrio completo, con parques, equipamientos, servicios, comercio y redes que dan lugar a una modalidad de vida urbana socialmente integrada y con buena calidad de vida.

Este desarrollo inmobiliario interior en la ciudad busca explícitamente vincularse con una política de desarrollo urbano, de vivienda y barrio que innove respecto de la mera expansión urbana. Al recuperarse este barrio y esta zona sur-poniente del Gran Santiago se agrega actividad urbana a la ciudad, estableciendo nuevas orientaciones de desarrollo inmobiliario y complementando esfuerzos ya emprendidos de renovación urbana en Santiago Centro, San Miguel, Ñuñoa y otros subcentros del Gran Santiago.

El Proyecto busca deliberadamente la construcción progresiva de un parque central de 50 hectáreas de carácter metropolitano, parques transversales que suman 20 hectáreas de carácter vecinal, la macroinfraestructura de redes de servicios y vial, simultáneamente con la construcción de las viviendas, buscando con ello una mejora en la calidad de vida de las 


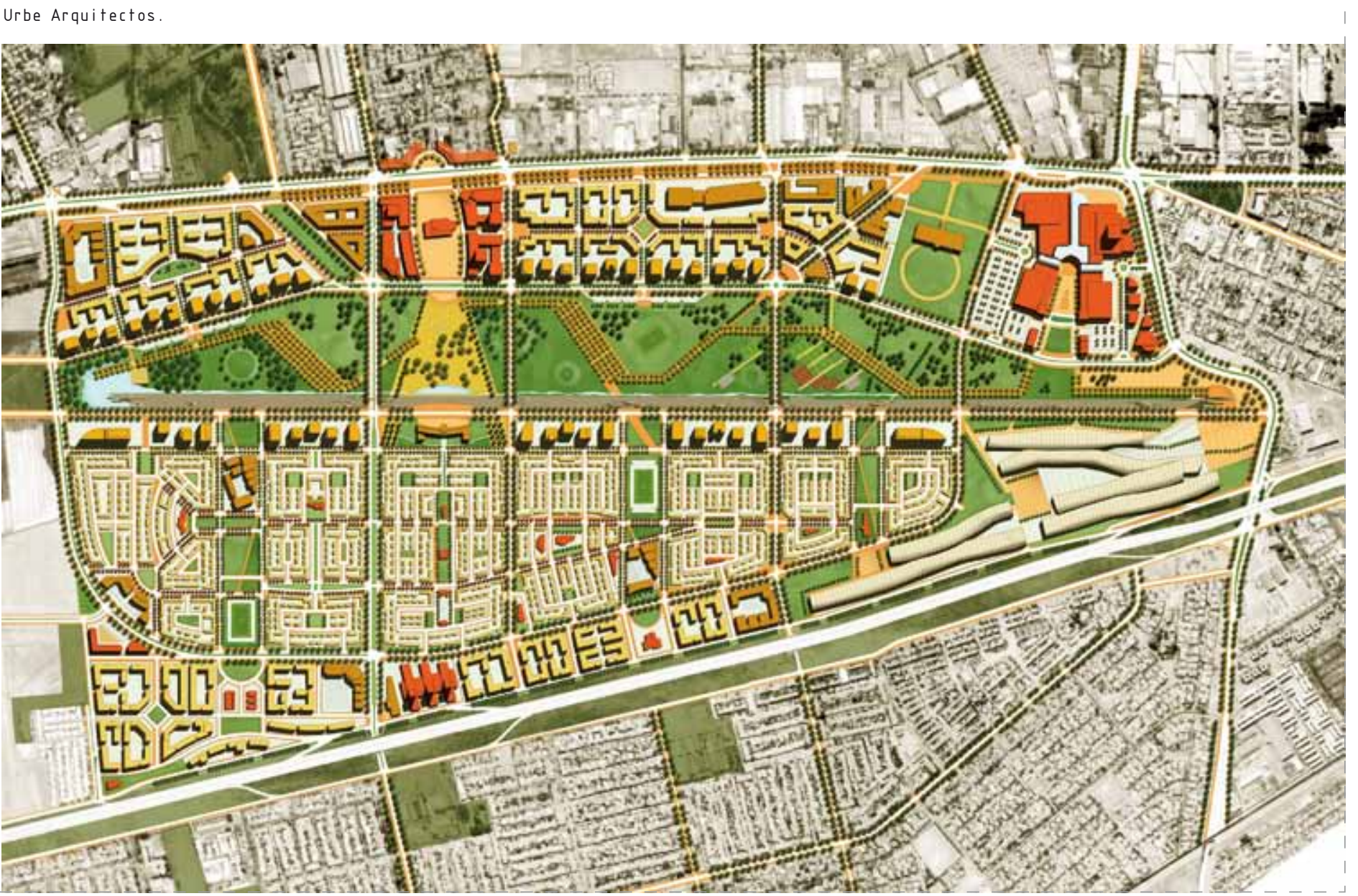

familias que vayan residiendo en un barrio de mejores estándares. Ello implica una relación vivienda-parque de nivel superior dentro del Gran Santiago.

Al 2010 buscamos el término de la construcción del parque y su mantenimiento con mecanismo propio, tiempo en el que se ha programado disponer de una cantidad importante de hectáreas de desarrollo inmobiliario y de la instalación plena de los equipamientos esenciales (especialmente educativos) y un Centro Consistorial. Un barrio integrado de equipamiento, servicios y comercio.

Esta "nueva ciudad" busca una arraigada integración social y mixtura social entre familias de distintos sectores de ingresos $y$, por lo tanto, busca una adecuada mezcla de soluciones habitacionales de distinto valor. Se debieran generar condiciones adecuadas de convivencia ciudadana, la que se expresará fundamentalmente en los amplios espacios de uso público que contempla el Proyecto. Ta como se desprende del proyecto ganador del Concurso Internacional de Ideas ${ }^{1}$, los parques y espacios públicos se constituirán en los elementos estructurantes del desarrollo del proyecto.

El Proyecto Portal Bicentenario ofrece un terreno único, de condiciones notables para el desarrollo de acciones inmobiliarias diversas, sobre la base de esquemas y diseños con razonables espacios públicos y socialmente integrados.

Principios Rectores Plan

Maestro Portal Bicentenario

Como segunda etapa se licita la realización de un plan maestro ${ }^{2}$, el que se concibe como la carta de navegación básica que permite establecer la coherencia y unidad entre los objetivos urbanos del proyecto, el modelo de gestión y el financiamiento público-privado. En él se definió los siguientes principios y criterios en el Ámbito Urbano Integral, establecidos en las Bases Técnicas planteadas:

- Imagen urbana unitaria, con identidad propia y responder a los fundamentos básicos que sustentan la Reforma Urbana, enfatizando la creación de un espacio urbano orientado a potenciar una alta calidad de vida a todos sus habitantes (residentes y usuarios).

- Diversidad e integración social, en la definición de los barrios residenciales, las zonas de equipamiento y los espacios públicos.

- Diversidad funcional, conformando un mix de usos, que permita un alto nivel de integralidad urbana, ofreciendo un amplio espectro de posibilidades en el ámbito residencial, de empleo, de servicios, de recreación y en cuanto al transporte público y privado.

En el que participan más de 70 propuestas nacionales y extranjeras. El Concurso fue ganado por los arquitectos

Cristián Ulloa y Rubén González, el año 2001.

Adjudicado a un Consorcio de Empresas lideradas por URBE Ltda. 

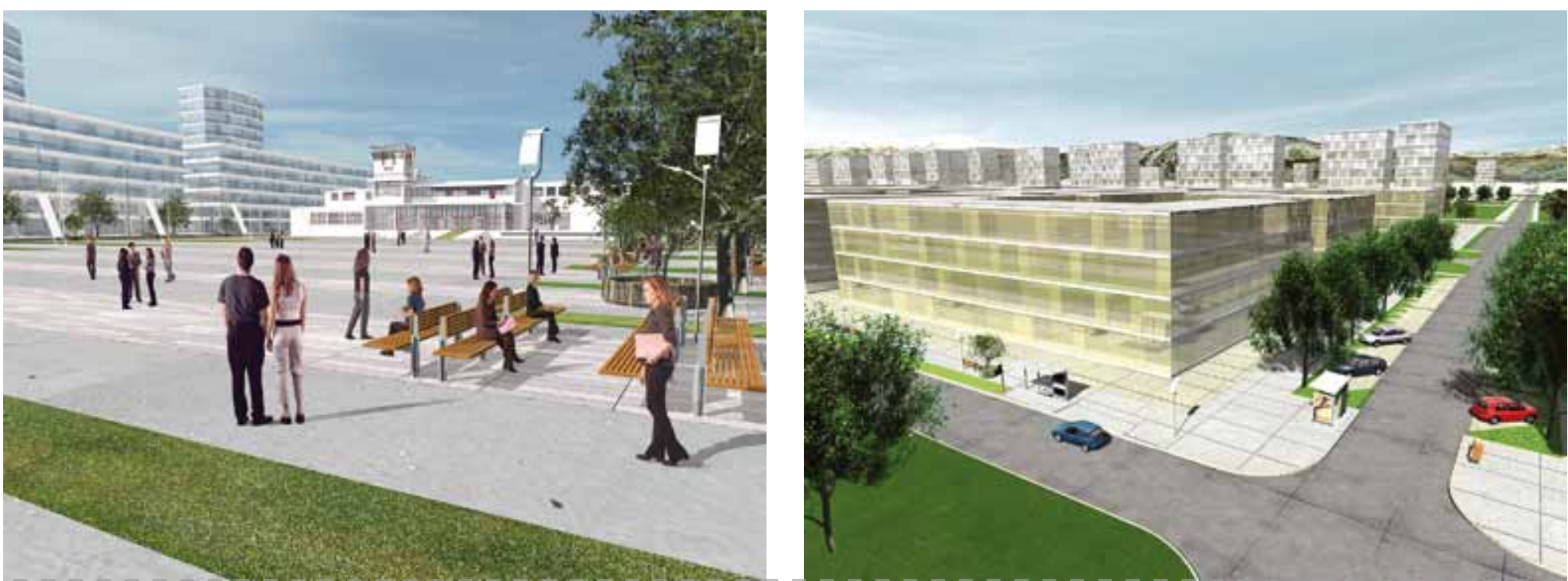

- Equilibrio Urbano, entre una alta intensidad de ocupación del terreno, caracterizado por su condición pericentral dentro del contexto Metropolitano y una generosa dotación de espacios públicos y áreas verdes que constituyan los principales elementos estructurantes y articuladores del espacio urbano.

- Integración espacial y funcional del área de proyecto respecto a su entorno inmediato, mediato y al resto de la ciudad, considerando desde su origen la continuidad de la trama vial, la dotación y calidad del transporte público, el acceso a equipamiento y las variables del transporte y su impacto como factores condicionantes del diseño y desarrollo del proyecto.

- Estrategia de desarrollo por etapas, otorgando las condiciones físicas y funcionales que le permitan adaptarse a los diversos escenarios de desarrollo, manteniendo las características fundamentales del proyecto.
- Velocidad de desarrollo y determinación de niveles de consolidación coherentes con el Plan Bicentenario, en el marco de posibilidades que otorga el mercado.

\section{Obras}

Actualmente, se encuentra en ejecución la obra de la primera etapa del parque central, y la primera etapa de la macrovialidad, la que permitirá el acceso a los primeros macrolotes que serán licitados este segundo semestre del 2006.

El Parque Portal Bicentenario, parque central de 50 hectáreas, apoyado en el trazado de la pista, recorre el terreno en toda su longitud, y a partir de este elemento organizador se desarrolla un sistema de parques transversales de escala vecinal y vías parque que complementan la función del parque central y ligan los distintos distritos del proyecto constituyendo una imagen de "ciudad parque". De esta manera, el sistema de espacio público en el proyecto -parques, plazas y calles- ocupa más de la mitad de la superficie total del terreno y su objetivo es convertirse en un fuerte atractivo para la futura población y en un aporte sustantivo para el resto de los habitantes de la comuna y del sector sur-poniente de Santiago.

El diseño del parque estuvo a cargo de los arquitectos Montealegre Beach, quienes obtuvieron el primer lugar de la licitación de diseño, actualmente se encuentra la primera etapa en ejecución, la que comprende la arborización, nivelación de terreno y sistema de regadío de las 50 hectáreas de parque, la construcción de la laguna, y el proyecto terminado de 8 hectáreas en la manzana que enfrenta al futuro centro cívico, que contempla el tratamiento de pavimentos, las plazas, la instalación de luminarias, mobiliario urbano y señalética. Esta etapa se concluirá en diciembre del 2006.

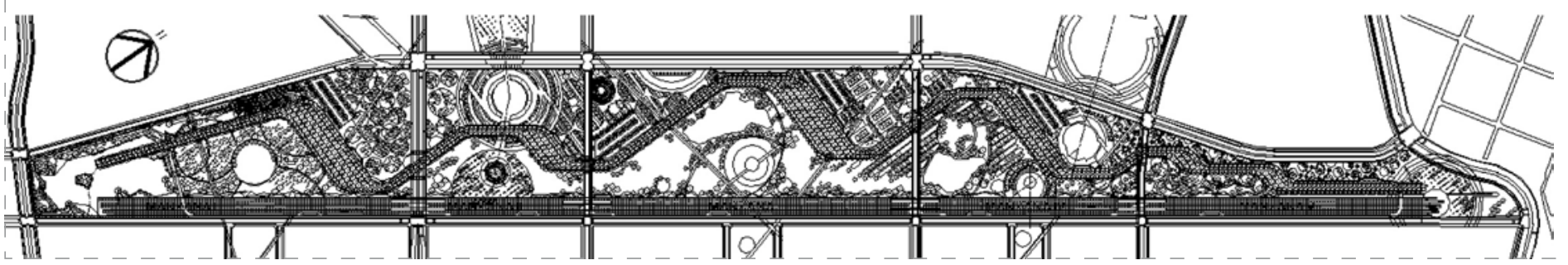

